\title{
Blind Multiuser Detection using Hidden Markov Models Theory
}

\author{
Carles Antón-Haro, José A.R. Fonollosa and Javier R. Fonollosa \\ Dept. of Signal Theory and Communications. Campus Nord. UPC. Edifici D-5 \\ c/ Gran Capità s/n. 08034 Barcelona (SPAIN) \\ Telf: +34-3-4016454 Fax: +34-3-4016447 e-mail: carles@gps.tsc.upc.es
}

\begin{abstract}
In this paper we present an adaptive algorithm based on the theory of Hidden Markov Models (HMM) which is capable of jointly detecting the users in a DSCDMA system. The proposed technique is near-far resistant and completely blind in the sense that no knowledge of the signature sequences, channel state information or training sequences is required for any user. In addition to this, an estimate of the signature of each user convolved with its physical channel impulse response (CIR), and an estimate of the background noise variance are provided once convergence is achieved (as well as estimated data sequences). At this moment, and using that CIR estimate, we can switch to any decisiondirected (DD) adaptation scheme.
\end{abstract}

\section{INTRODUCTION}

It's well known that no high-speed band-limited digital communication can be carried out without the help of an equalizer. Conventional approaches to the adjustment of this equalizer require the transmission of a training sequence (i.e. known a priori by the receiver and the transmitter), which provides an accurate initial estimate for the equalizer taps; afterwards, slighter adjustments can be made on a decisiondirected (DD) basis to adapt this first estimate to the, almost always, changing environment. Of course, the transmission of these training sequences, when possible, brings down the capacity of the system. For that reason, there is an increasing interest in blind equalizers $[1,2,3]$ which deal with the tap adjustment without training sequences (i.e. blindly).

On the other hand, CDMA techniques have become one of the most useful strategies in order to provide multiple access in digital communications. In [4], a Viterbi-based blind algorithm is proposed to jointly perform channel estimation and sequence detection in a CDMA environment. Nevertheless, modeling the received signal as a HMM [5] allows us to make use of the complete theory developed for these models. For example, the Baum\&Welch (BW) algorithm was proposed in [6] to estimate the parameters of the channel and the characteristics of the modulation in a single-user case. This algorithm is known to lead, at least, to a local maximum of the likelihood function $[5,6]$, what is not guaranteed by the Viterbi algorithm (VA).

This paper is devoted to build a signal model and to include several modifications to this previously proposed $\mathrm{BW}$ algorithm, to adapt it to a multiuser CDMA system.

\section{SIGNAL MODEL}

We consider the general asynchronous multiple-access channel model. The received signal is given by:

$$
r(t)=\sum_{n} \sum_{k=1}^{K} b_{k}[n] h_{k}(t-n T, t)+\sigma w(t)
$$

where $h_{k}(t-n T, t)$ is the overall complex channel impulse response of user $k$ given by the convolution of its signature sequence, physical channel and the receiving filter responses. It incorporates the amplitude, the Doppler frequency deviation and the delay for user $k$, and its duration is assumed to be no longer than $L$ symbol periods. The total number of active users is $K$ and their transmitted data sequences are binary independent symbols $b_{k}[n] \in\{-1,1\}$. The symbol rate is $1 / T$ and $w(t)$ is normalized AWGN. The multiple-access channel is sampled at a rate $f_{s}=1 / T_{s}=M / T$ to derive the discrete vector sequence $\mathbf{r}[n]$ :

$$
\mathbf{r}[n]=\left[r(n T), \ldots, r\left(n T+(M-1) T_{s}\right)\right]^{T}
$$

where ${ }^{T}$ denotes transpose operation. The observation $\mathbf{r}[\mathrm{n}]$ can be modeled as a $M$-length vector, probabilistic function of a state vector $s[n]$ :

$$
\mathbf{r}[n]=\mathbf{H}[n] \mathbf{s}[n]+\mathbf{w}[n]
$$

Since at any given time a maximum of $L$ symbols for each user affect the observation, there are $N=2^{L K}$ possible state vectors corresponding to all combinations of $L$ binary symbols of the $K$ active users. We denote each of the possible states as the $K L$-length vector $\mathbf{s}_{j}$,

$$
\mathbf{s}_{j} \in \mathbf{S}=\left\{\mathbf{s}_{1}, \mathbf{s}_{2}, \ldots, \mathbf{s}_{N}\right\}
$$

such that,

$$
\begin{gathered}
\mathbf{s}_{j}=\left[\mathbf{s}_{j 1}{ }^{T}, \ldots, \mathbf{s}_{j K}{ }^{T}\right]^{T} \\
\mathbf{s}_{j k}=\left[s_{j k}^{(0)}, \ldots, s_{j k}^{(L-1)}\right]^{T}, \quad s_{j k}^{(l)} \in\{-1,1\}
\end{gathered}
$$

The actual state at time instant $n T$ is denoted by $\mathbf{s}[n], \mathbf{s}[n] \in \mathbf{S}$. The $(M x K L)$ matrix $\mathbf{H}[n]$ depends on the overall discrete impulse responses for all users, denoted by matrices $\mathbf{H}_{k}[\mathrm{n}]$

$$
\mathbf{H}[n]=\left[\mathbf{H}_{1}[n], \ldots, \mathbf{H}_{K}[n]\right]
$$

Each of these matrices incorporates a vector response for the $L$ symbols that may be present in the observation due to multipath propagation,

$$
\mathbf{H}_{k}[n]=\left[\mathbf{h}_{k 0}[n], \ldots, \mathbf{h}_{k(L-1)}[n]\right]
$$

and, finally, the resulting signature for each user and symbol:

$$
\mathbf{h}_{k l}[n]=\left[\begin{array}{c}
h_{k l}((n+l) T, n T) \\
\vdots \\
h_{k l}\left((n+l) T+(M-1) T_{s}, n T\right)
\end{array}\right]
$$

The noise is characterized as the $M$-length vector

$$
\mathrm{w}[n]=\sigma\left[w(n T), \ldots, w\left(n T+(M-1) T_{s}\right)\right]^{T}
$$




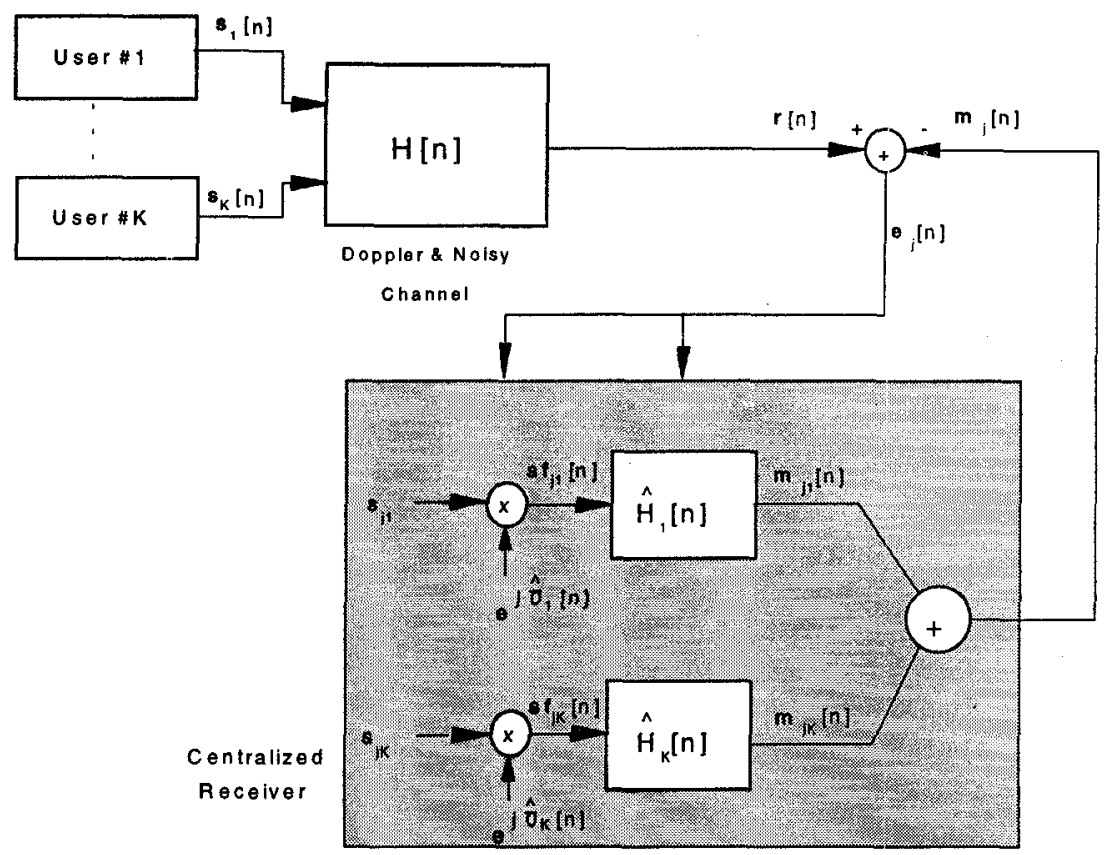

Fig. 1: System model.

As a consequence of this modeling, we obtain a description of the sequence $\mathbf{r}_{D}=(\mathbf{r}[1], \mathbf{r}[2], \ldots, \mathbf{r}[D])^{T}$ as a first order $\operatorname{HMM}(\lambda)[5]$ with the following features:

1. The number of states is $N=2^{L K}$, i.e., the number of distinct inputs that the system may have.

2. The probability density function of the observation $\mathbf{r}$ conditioned on a given state channel $\mathbf{s}_{j}$ is

$$
\mathrm{p}\left(\mathbf{r}[n] \| \mathbf{s}_{j}\right)=\frac{1}{\sigma \sqrt{2 \pi}} \exp \left(-\frac{\left\|\mathbf{r}[n]-\mathbf{m}_{j}[n]\right\|^{2}}{2 \sigma^{2}}\right)
$$

where

$$
\mathbf{m}_{j}[n]=\mathbf{H}[n] \mathbf{s}_{j}, \quad 1 \leq j \leq N
$$

3. The state transition probability distribution is

$$
\begin{gathered}
\mathbf{A}=\left\{\mathrm{a}_{i j}\right\}, \quad 1 \leq i, j \leq N \\
\mathrm{a}_{i j}=\mathrm{P}\left(\mathrm{s}[n+1]=\mathbf{s}_{j} \mid \mathrm{s}[n]=\mathbf{s}_{i}\right)= \\
\left\{\begin{array}{cc}
\mathrm{P}\left(s_{j 1}^{(0)} \cap \ldots \cap s_{j K}^{(0)}\right) & \text { if } s_{j k}^{(l)}=s_{i k}^{(l-1)}, l=1 \ldots L-1, \\
0 \quad & k=1 . . K
\end{array}\right. \\
\text { where } s_{j k}(l) \in\{-1,1\} \text { denotes the } l^{\text {th }} \text { symbol (bit) in } \mathbf{s}_{j k}
\end{gathered}
$$

We will assume that $K$ is known and $L$ is either known or can be upper bounded. Then, we can follow standard HMMbased approaches to determine the unknown parameters of our model: (1) $\mathbf{M}$, the $M x N$ means matrix given by:

$$
\mathbf{M}[n]=\left[\mathbf{m}_{1}[n], \ldots, \mathbf{m}_{N}[n]\right]
$$

which stands for the noise-free ISI-corrupted received multiuser signal corresponding to each state of the model (or, equivalently to this parameter, the overall multiuser channel impulse responses $\mathbf{H}[n]$ ); (2) $\sigma^{2}$, the AWGN variance; and (3) $\mathrm{P}_{\mathrm{a}}=(\mathrm{P}(-1), \mathrm{P}(1))^{\mathrm{T}}$, the probability distribution of the symbols. Note that, according to Fig. 1, all $K$ users contribute to every means vector according to:

$$
\mathbf{m}_{j}[n]==\sum_{k=1}^{K} \mathbf{m}_{j k}[n]
$$

We will assume, without loss of generality, that symbols are equally likely. This allows us to simplify the derivations and the expressions for the proposed estimation methods.

\section{BLIND IDENTIFICATION AND DETECTION ALGORITHM}

We will concentrate in a recursive version of the algorithm called ABW (Adaptive Baum\&Welch) [6] that is iterated at the symbol rate. First of all, we will obtain $\gamma_{i}[n]$, the probability of being in state $s_{i}$ at time instant $n$, given the observed sequence, $\mathbf{r}_{\mathrm{D}}$, and the model $\lambda$. The ForwardBackward algorithm $[5,6]$ provides a computationallyefficient way to compute this variable. Secondly, and taking into account $\gamma_{i}[n]$, we will reestimate the parameter set of the model $\left(\mathbf{M}[n+1], \quad \sigma^{2}[n+1]\right)$ using the $\mathrm{BW}$ reestimation method. For this purpose, the BW reestimation formulas [5] are rewritten in a recursive manner so that the algorithm can track slow variations in the parameters of the model. When the channels, as in our case, are modeled as linear FIR systems, instead of adapting the means, we can directly adapt the CIR estimate. In the framework of the steepest-descent algorithm for system identification we have (see Fig.1):

$$
\hat{\mathbf{H}}[n]=\hat{\mathbf{H}}[n-1]+\mu_{h} \mathrm{E}\left[\mathbf{e}[n] \mathbf{s} \mathbf{f}[n]^{H}\right]
$$

where

$$
\begin{gathered}
\mathbf{e}[n]=\mathbf{r}[n]-\mathbf{m}[n] \\
\mathbf{s f}[n]=\left[\mathbf{s f}_{1}[n]^{T}, \ldots, \mathbf{s f}_{K}[n]^{T}\right]^{T}
\end{gathered}
$$

and ${ }^{H}$ stands for conjugate transpose. In our blind environment, the expectation in the gradient term will be computed in the following manner. 


$$
\begin{aligned}
\hat{\mathbf{H}}[n] & =\hat{\mathbf{H}}[n-1]+\mu_{h} E_{r_{b}, \lambda}\left[\mathbf{e}[n] \mathbf{s} \mathbf{f}[n]^{H}\right]= \\
& =\hat{\mathbf{H}}[n-1]+\mu_{h} \sum_{j=1}^{N} \gamma_{j}[n] \mathbf{e}_{j}[n] \mathbf{s} \mathbf{f}_{j}[n]^{H}
\end{aligned}
$$

where:

$$
\begin{array}{r}
\mathbf{e}_{j}[n]=\mathbf{r}[n]-\mathbf{m}_{j}[n-1], \quad 1 \leq j \leq N \\
\mathbf{s f}_{j}[n]=\mathbf{s}_{j} \otimes \\
{\left[\exp \left(j \hat{\theta}_{1}[n]\right), \ldots, \exp \left(j \hat{\theta}_{1}[n]\right), \ldots,\right.} \\
\left.\ldots, \exp \left(j \hat{\theta}_{K}[n]\right), \ldots, \exp \left(j \hat{\theta}_{K}[n]\right)\right] \\
\leftarrow \quad-\text { times } \quad \rightarrow
\end{array}
$$

and $\otimes$ stands for element-by-element product. Two advantages show up when using this approach: (1) we obtain an estimate for the CIRs at each time instant and, (2) since the Forward-Backward iteration should operate with the means matrix, it makes possible to incorporate additional linear constraints between $\mathbf{M}$ and $\mathbf{H}$ :

$$
\mathbf{M}[n]=\hat{\mathbf{H}}[n] \mathbf{S} \mathbf{f}[n]
$$

where:

$$
\mathbf{S f}[n]=\left[\mathbf{s f}_{1}[n], \ldots, \mathbf{s f}_{N}[n]\right]
$$

The AWGN variance estimate is updated according to:

$$
\hat{\sigma}^{2}[n]=\hat{\sigma}^{2}[n-1]+\mu_{s}\left(\sum_{j=1}^{N} \gamma_{j}[n]\left\|\mathbf{e}_{j}[n]\right\|^{2}-\hat{\sigma}^{2}[n-1]\right)
$$

Finally, data detection, is performed following an individually most likely state (IMLS) criterion .

\section{A. Inclusion of a blind PLL}

As depicted in Fig.1 and equation (21), we compensate Doppler frequency shifts separately with the inclusion of a blind PLL. Adopting such strategy increases the robustness of the receiver, in particular in environments (such as third generation personal communication systems) where large frequency deviations appear as a consequence of high speed of the mobiles.

According to [7], we introduce a second order DPLL into the process of joint channel estimation and data detection. The update equation is given by:

$$
\hat{\theta}_{k}[n+1]=\hat{\theta}_{k}[n]+K_{k 1} \phi_{k}[n]+K_{k 2} \sum_{m=0}^{n} \phi_{k}[m]
$$

where $K_{k 1}$ and $K_{k 2}$ are proportional and integral tracking constants respectively. The term $\phi_{k}[n]$ is the instantaneous estimate of the MSE gradient w.r.t. the phase estimate for user $k$ :

$$
\phi_{k}[n]=\frac{\partial\|\mathbf{e}[n]\|^{2}}{\partial \theta_{k}}=\operatorname{Im}\left\{\mathbf{e}[n]^{H} \mathbf{m}_{k}[n]\right\}
$$

Where null crosscorrelation between transmitted data sequences has been assumed. Again, in our blind environment, $\mathbf{m}_{k}[n]$ is replaced by its estimate given the received data and the model, namely:

$$
\begin{aligned}
\phi_{k}[n] & =E_{\mathbf{r}_{p}, \lambda}\left[\operatorname{Im}\left\{\mathbf{e}[n]^{H} \mathbf{m}_{k}[n]\right\}\right]= \\
& =\operatorname{Im}\left\{\sum_{i=1}^{N} \gamma[n] \mathbf{e}[n]^{H} \mathbf{m}_{i k}[n]\right\}
\end{aligned}
$$

\section{B. Summary of the algorithm}

To sum up, the whole algorithm states as follows:

- Initialization of the algorithm:

Set initial values for $\hat{\boldsymbol{\theta}}_{k}(k=1 . . K), \hat{\mathbf{H}}, \hat{\sigma}^{2}$.

- Iteration for all received data:

for $\mathrm{n}=1 . . \mathrm{D}$,

- Estimation of $M[n]$ by means of the additional linear constraint. (Eq. 22)

- BW iteration:

* Compute the variable $\gamma_{\mathrm{i}}[n] \quad(\mathrm{i}=1 . . \mathrm{N})$

* Data detection (IMLS criterion)

* Reestimation of the parameter set:

$$
\hat{\theta}_{k}[n+1], \hat{\mathbf{H}}[n+1] \hat{\sigma}^{2}[n+1]
$$

end;

\section{AVOIDING LOCAL MAXIMA}

As mentioned previously, BW-based algorithms lead, at least to a local maximum of the likelihood function. When dealing with CDMA signals, those maxima appear as a consequence of two fairly independent phenomena [7]: severe Intersymbol Interference, and multiuser structure of the signal resulting from the near-far effect. The first circumstance rarely occurs in most practical radio channels in which ISI spans a very few symbols. Therefore, we will concentrate on analyzing and solving the problems in our algorithm associated to the near-far effect. When a multiuser signal is affected by the near-far effect, the algorithm tends to split the CIR estimate of the user(s) of greatest magnitude among the weakest(s) ones since it locally maximizes the likelihood function. Nevertheless, this extent can be easily detected since, in those cases, estimated data sequences for the involved users are identical (up to a sign change). The strategy employed in the present paper to overcome this problem was suggested in [4]. It consists in checking, every $S$ symbols, the crosscorrelation of the estimated data sequences between users (evaluated for $2 \cdot L+1$ lags). Estimated CIRs for the number of users with matching data sequences, say $K_{m}$, are added up and arbitrarily assigned to one of them whereas those of the remaining $K_{m}-1$ are set to the initial value. Of course, the same operation must be performed with the rest of parameters being estimated, in this case, Doppler frequency shift. Note that this approach leads to an unpredictable assignment of the estimated CIRs to the users.

A final remark. If, at any moment, the estimate of noise level is higher than the energy of any user's CIR estimated so far, the system tends to converge towards the trivial solution $(H \equiv 0)$, i.e. considering the whole signal as noise. This might happen if the system were suddenly disturbed, for instance after coherence compensations. Therefore, we impose the additional restriction that, at any time instant, the estimate for the noise variance can not be greater in magnitude than the energy of any of the already-estimated CIRs.

\section{SIMULATION RESULTS}

The system under study employs a BPSK modulation scheme. The received signal is sampled at a rate of 1 sample/chip although the identification algorithm operates at the symbol rate. For all simulations, the constants in the 
update equations were set to: $\mu_{h}=2 \cdot 10^{-2}, \mu_{s}=4 \cdot 10^{-2}, K_{k l}=10^{-3}$ and $K_{k 2}=10^{-4}$. Several tests were performed for power difference between users up to $30 \mathrm{~dB}$, and for SNR -for the weakest user- ranging from 6 to $12 \mathrm{~dB}$. Distortion due to Doppler frequency shifts has been considered too. Unless stated otherwise, the results are averaged over 50-run simulations.

First, a case of $K=4$ users received with similar power is considered. Figure 2 shows the evolution of the estimated CIR (real part) in a single-run simulation. Note that, despite of the joint detection strategy, users are extracted sequentially -within the first 300 symbols- in accordance with their received amplitudes. Sudden jumps in the estimates are due to coherence compensations. The averaged estimation noise for CIRs corresponding to each user (learning curves) is depicted in Fig. 3.

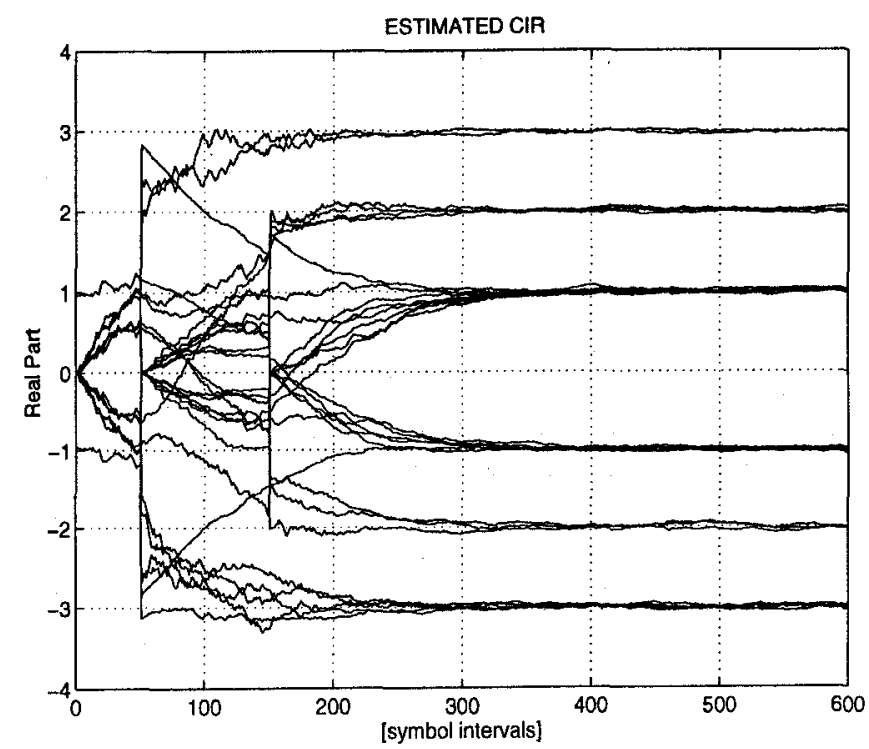

Fig. 2: Evolution of CIR estimate (real part, single-run test). Users' amplitudes: $3,2,1,1 . S N R=12 \mathrm{~dB}$.

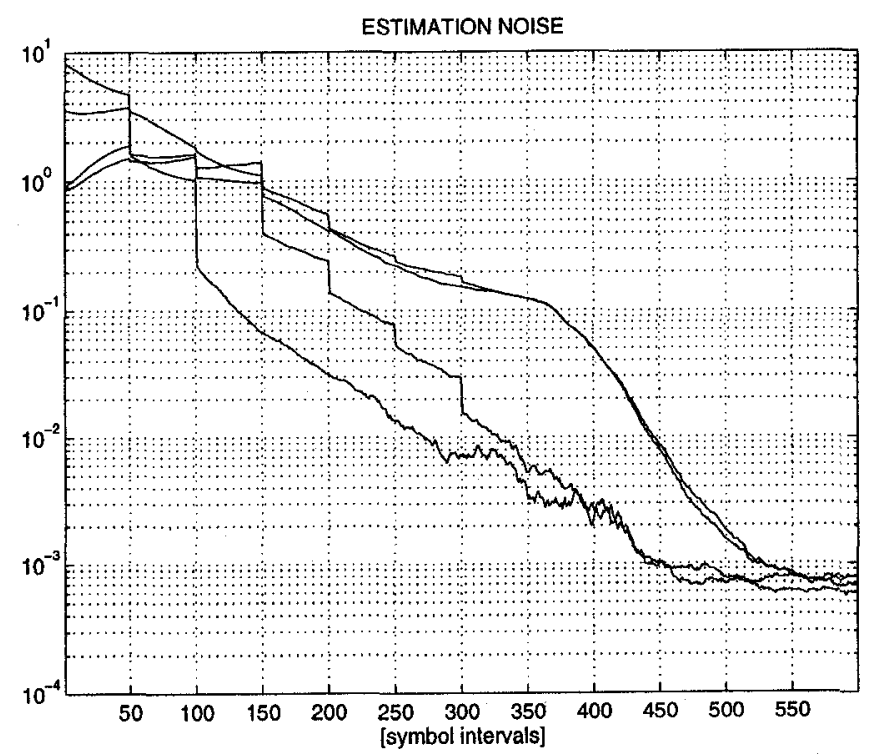

Fig. 3: Learning curves for different users. Amplitudes: 3, 2, 1, 1. $\mathrm{SNR}=12 \mathrm{~dB}$.

Figure 4, is devoted to plot the evolution of the AWGN variance estimate. It asymptotically attains the true value $\left(\sigma^{2}=0.063\right)$, as long as the contribution from the estimation noise goes to zero.

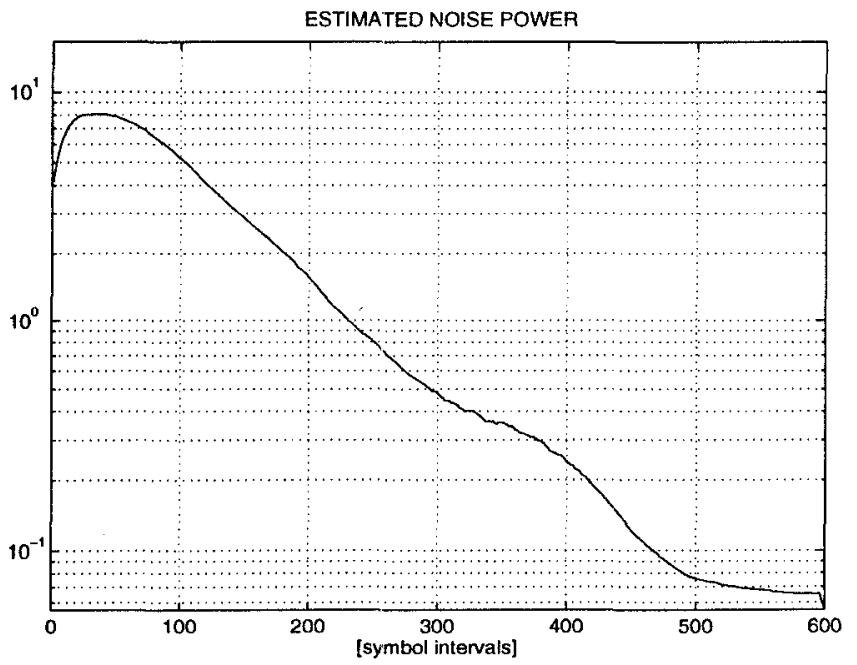

Fig. 4: Evolution of the AWGN variance estimate. Amplitudes: 3, 2, $1,1 . S N R=12 \mathrm{~dB}$.

Behaviour when facing near-far effect is depicted in Figs. 5-6. A maximum of (approximately) $30 \mathrm{~dB}$ power difference between users is considered and, despite of this, signals are extracted within a few hundred symbols as happened before.

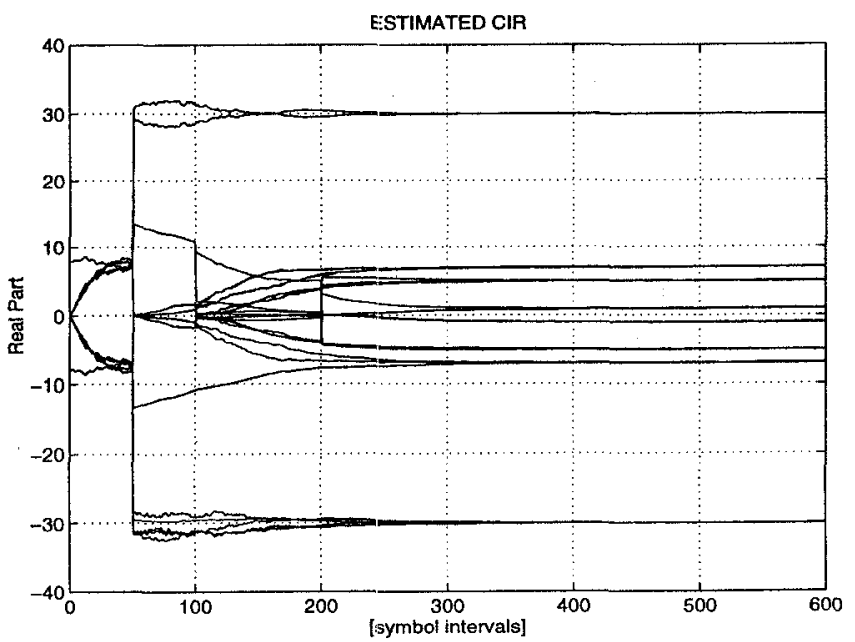

Fig. 5: Evolution of CIR estimate (real part, single-run test) with near-far effect. Users' amplitudes: $30,7,5,1$. SNR=12 dB.

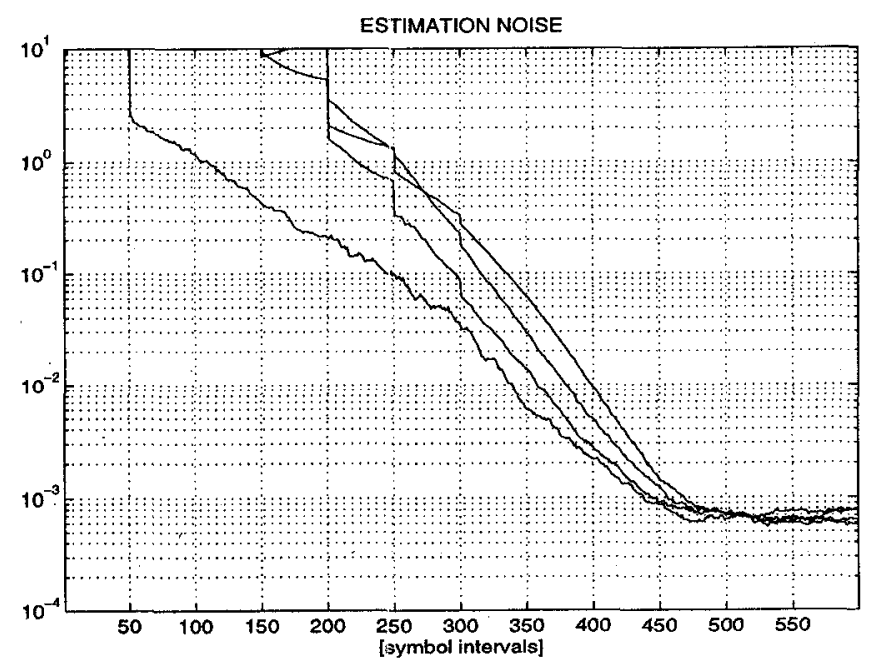

Fig. 6: Learning curves for different users with near-far effect. Users' amplitudes: $30,7,5,1$. SNR=12 dB.

Other tests were performed for a difference of $40 \mathrm{~dB}$; the algorithm also succeeded in separating users. 
In Fig. 7, the system is tested in a noisy environment. We observe that users separation is also achieved despite of low SNR, 6dB. Since the CIR is obtained as an average for all channel states, such algorithm is specially robust to noise. Note, however, that estimation noise is higher now.

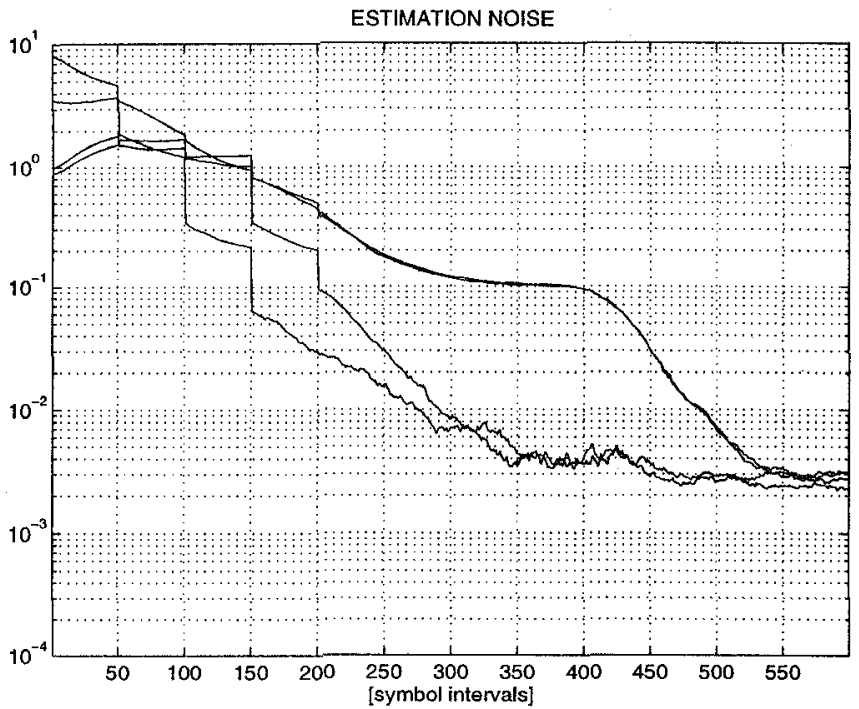

Fig. 7: Learning curves for different users in a noisy environment. Users' amplitudes: $3,2,1,1$. SNR=6 dB.

Finally, the system is checked in the presence of large amounts of Doppler frequency shift: a phase shift of about 20 rads within 600 symbols. In Fig. 8 (one-run plot), we observe that the blind PLL is capable of tracking the evolution of such phase shift (except for an additive constant), thus allowing the $\mathrm{BW}$ algorithm to converge. In general, phase shift tracking is split between the blind PLL and the CIR estimates since the latter can track slow changes of such parameter too.

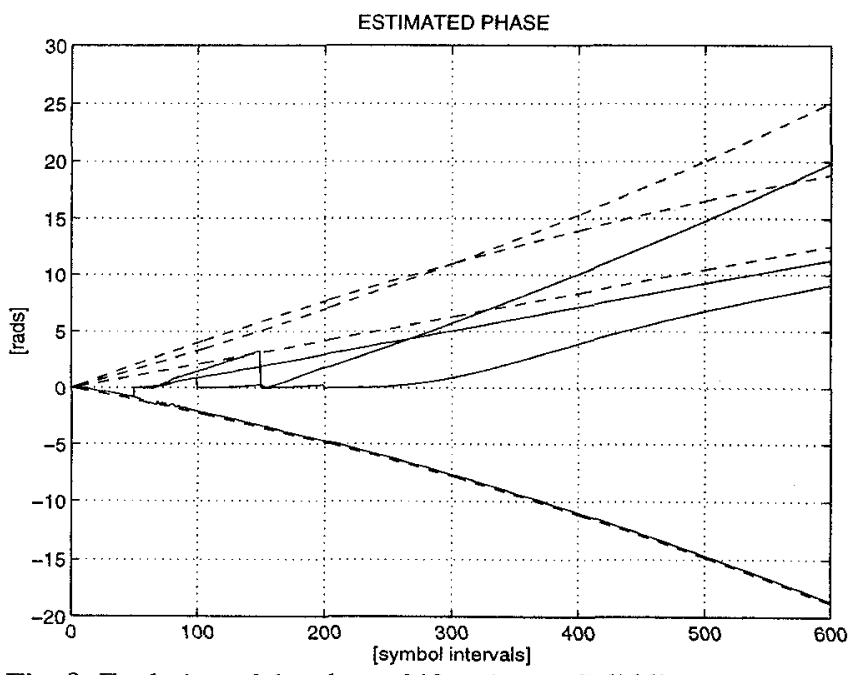

Fig. 8: Evolution of the phase shift estimate. Solid lines correspond to the estimate; dash-dot lines to the true value. Users' amplitudes: $10,7,5,1 . \mathrm{SNR}=12 \mathrm{~dB}$.

\section{CONCLUSIONS AND FUTURE WORK}

In this paper, a joint detection algorithm for DS-CDMA signals has been presented. In the proposed scheme, users are extracted sequentially according to decreasing power order. The estimate of the convolution of each user's signature with the physical channel is recursively updated using the steepest-descent approach. Nevertheless, since the receiver operates blindly, training sequences are replaced by estimates of the transmitted data based on the received signal and the present parameter set of the HMM. Such parameter set is adjusted with the help of the forward-backward iteration and the Baum\&Welch (BW) reestimation procedure. The CIR estimate incorporates frequency shift compensation with the inclusion of a blind PLL. The motivation for adopting such strategy is increasing the robustness of the receiver, in particular in environments (such as third generation personal communication systems) where large frequency deviations appear as a consequence of high speed of the mobiles. The resulting algorithm is wellsuited for the startup period of a system in which, after convergence, we can switch to any less computationallyintensive decision-directed adaptation method.

Undergoing research focuses on the inclusion of array observation in the present framework, comparison with other blind detection techniques and computational load reduction. Further validation is also due by considering synthetic signals generated by standard test channels (such as those proposed in the GSM recommendation [8]) or, otherwise, real data.

\section{ACKNOWLEDGEMENTS}

This work has been partially supported by the Catalan Foundation for Research, the CIRIT (GRQ93-3021), and the CICYT of Spain.

\section{REFERENCES}

[1] J.G Proakis and C.L. Nikias, "Blind Equalization", Proc. SPIE Adaptive Signal Processing 1991, Vol. 1565, pp. 76-87, 1991.

[2] J. Shynk et al., "A Comparative Performance Study of Several Blind Equalization Algorithms", Proc. SPIE Adaptive Signal Processing 1991, Vol. 1565, pp. 102-117, 1991.

[3] M. Ghosh and C.L. Weber, "Maximum Likelihood Blind Equalization", Proc. SPIE Adaptive Signal Processing 1991, Vol. 1565, pp. 188-195, 1991.

[4] J.R. Fonollosa, J.A.R. Fonollosa, Z. Zvonar and J. Vidal, "Blind Multiuser Identification and Detection in CDMA Systems", Proc. IEEE ICASSP'95, pp. 1876-1879, May 1995.

[5] L. Rabiner, "A Tutorial on Hidden Markov Models and Selected Applications in Speech Recognition", Proc. IEEE, Vol. 77, No. 2, pp. 257-286, Feb. 1989.

[6] J.A.R. Fonollosa and J. Vidal, "Application of Hidden Markov Models to Blind Channel Characterization and Data Detection", Proc. IEEE Int. Conf. Acoust. Speech and Signal Processing, pp. 185-188, Australia, April 1994.

[7] Z. Zvonar, D. Brady and J. Catipovic, "An Adaptive Linear Multiuser Receiver for Deep Water Acoustic Local Area Networks", Proc. IEEE ICASSP'94, Vol. II, pp. 389-392, April 1994

[8] R. Steele, "Mobile Radio Communications", Pentech Press Publishers, London 1992. 